Supporting Information

\title{
Metal-Free Transparent Three-Dimensional Flexible Electronics by Selective Molecular Bridges
}

Wei-Shuo Chang, $†$ Ta-Sheng Chang, $†$ Chang-Ming Wang, and Wei-Ssu Liao*

Department of Chemistry, National Taiwan University, Taipei 10617, Taiwan

$†$ These authors contributed equally

*To whom correspondence should be addressed: wsliaochem@ntu.edu.tw (W.S.L.) 


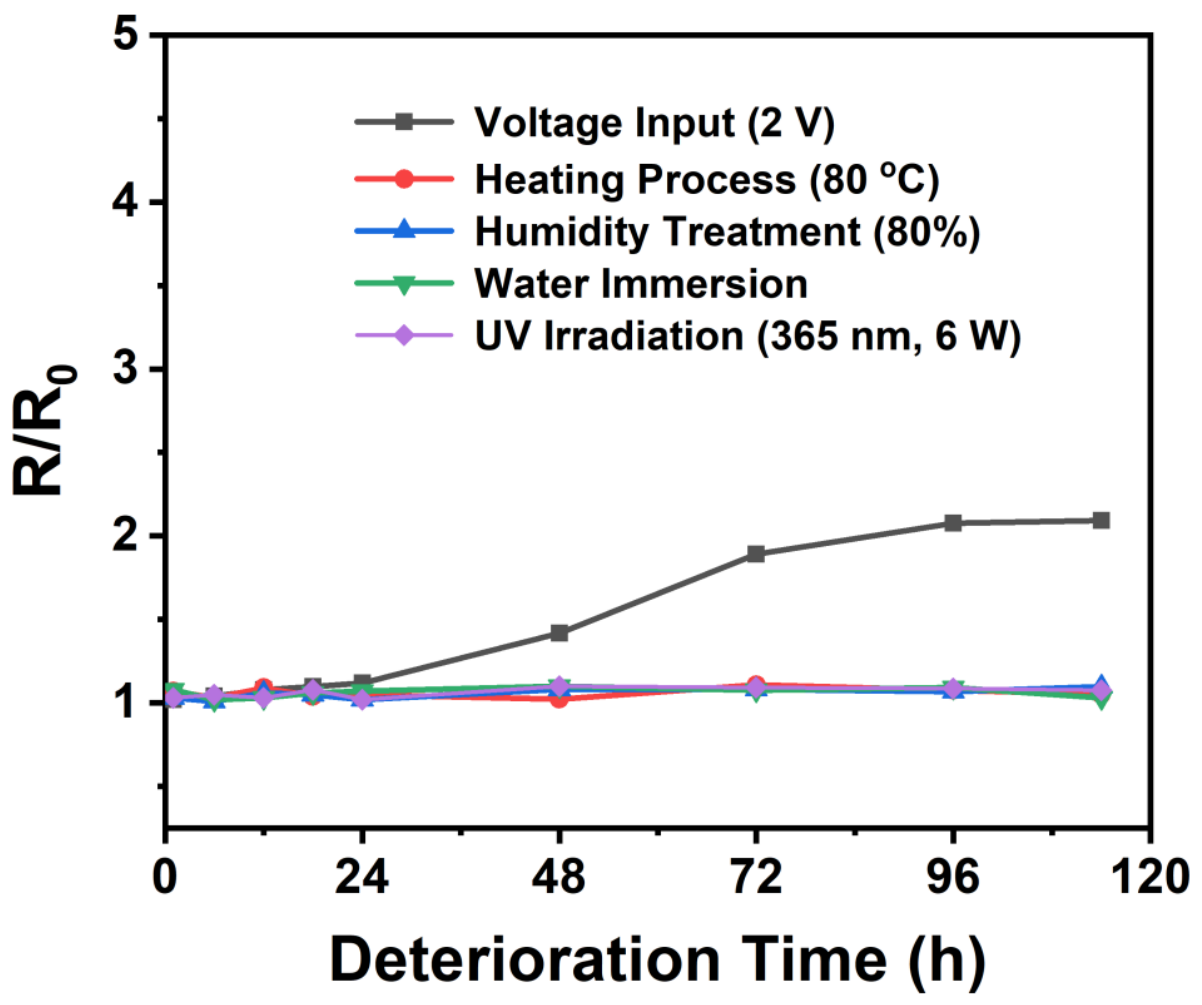

Figure S1. Changes in sheet resistance with time when a PEDOT:PSS/PVA/PET film is treated under five different environmental conditions. 


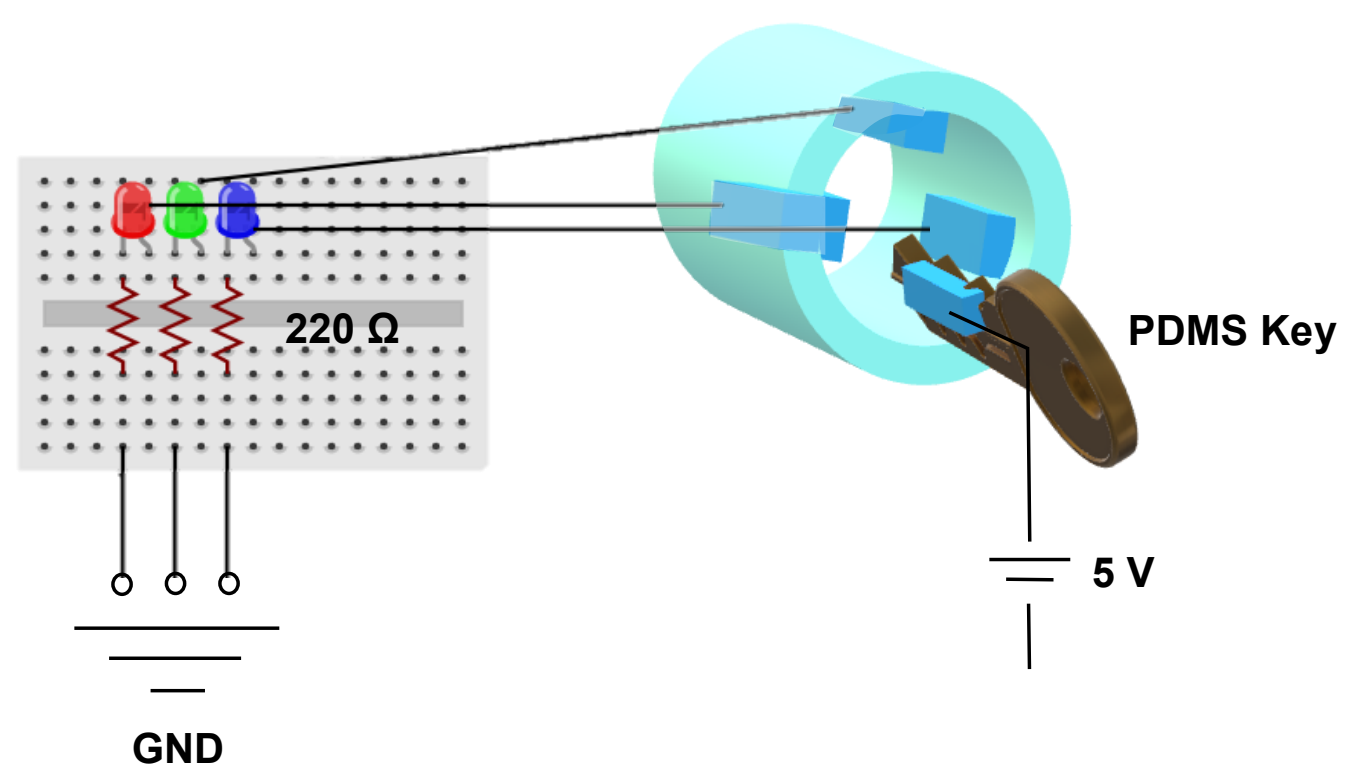

Figure S2. Circuit design of transparent three-dimensional flexible door lock and key pair electronics by PEDOT:PSS/PVA/PET films. 
(A) Folding circuit
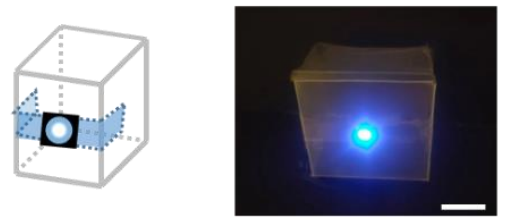

(C) Interlacing circuit

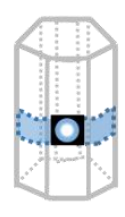

(B) Splicing circuit

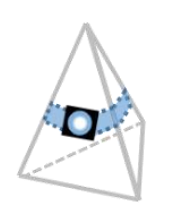

(D) Braiding circuit
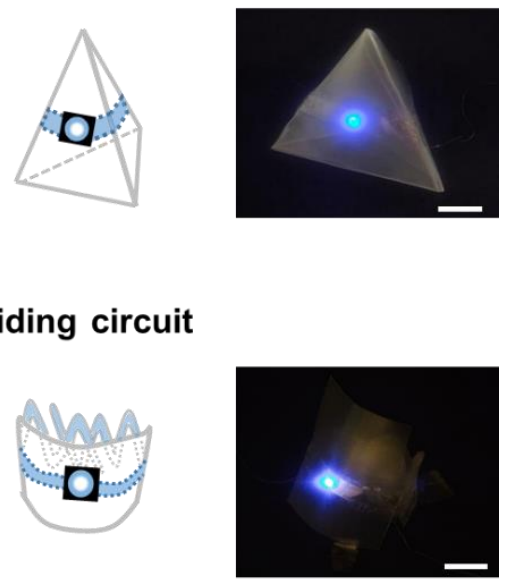

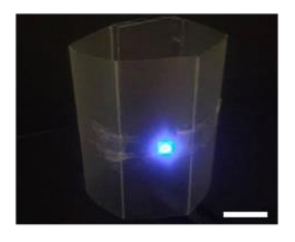

PET Film

LED

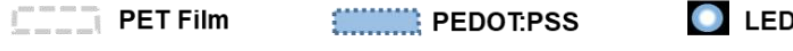

Figure S3. Electric circuit visual confirmation of flexible origami electronics with diverse circuit connection designs by LED illumination including (A) folding, (B) splicing, (C) interlacing, and (D) braiding. The scale bars are $1 \mathrm{~cm}$. 
(A)

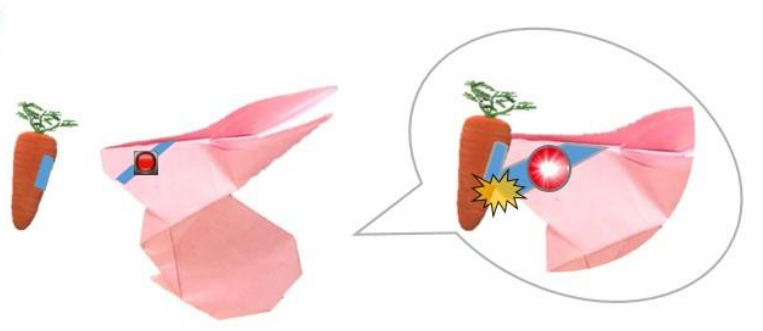

(B)

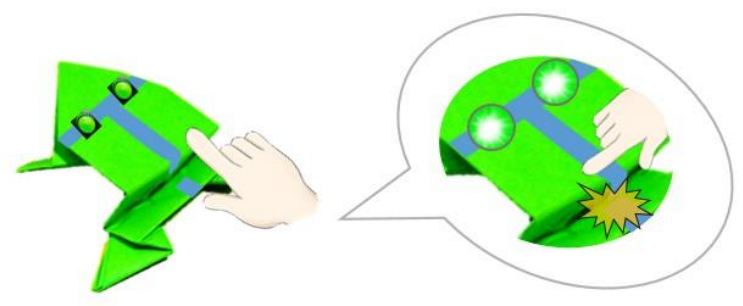

(C)
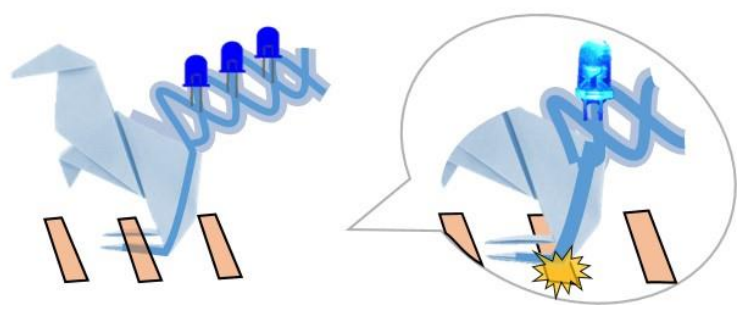

Figure S4. Sophisticated origami design schematic illustrations for human-machine interfacial motions including (A) rabbit and carrot touch pair, (B) pressure-stimulated jumping frog, and (C) directional moving dinosaur. 